\title{
SENSITIVITY OF A GALVANOMETER AS A FUNCTION OF ITS RESISTANCE
}

\author{
By H. B. Brooks
}

\section{ABSTRACT}

Maxwell derived a theorem which states that for the maximum deflection of a galvanometer connected to a given external circuit containing a given electromotive force the ratio of galvanometer resistance to external resistance should be equal to the ratio of the diameter of the wire in the galvanometer coil (before insulation) to the diameter including the insulation. Ayrton and Perry later stated that the galvanometer resistance should be equal to the external resistance. This widely quoted statement differs from Maxwell's because Maxwell assumed an unchanging thickness of insulation, Ayrton and Perry a thickness varying directly as the diameter of the bare wire.

The present paper demonstrates that while both statements are correct for their respective assumptions, the sensitivity function in each case is very flat in the vicinity of the maximum; that aside from considerations of damping the user has a wide range of choice of galvanometer resistance with relatively small loss of sensitivity, and can in fact sometimes obtain much better sensitivity with moving-coil galvanometers (when critical damping is considered) by departing from the theoretical optimum value of resistance. A general curve of the sensitivity function for the Ayrton-Perry assumption is given with a similar curve for a particular case for the Maxwell assumption. The performance of four lines of commercial galvanometers is shown by plotted points.

The constructional limitations and those related to damping which must be considered in applying the conclusions of the paper are outlined, and the practical advantages of the facts brought out, to both maker and user, are briefly summarized.

\section{CONTENTS}

Page

I. Introduction

297

II. Sensitivity of the moving-magnet galvanometer

III. Sensitivity of the moving-coil galvanometer

IV. Sensitivity for conditions widely different from the optimum

V. Damping limitations of the moving-coil galvanometer.

VI. Constructional limitations

VII. Practical considerations

\section{INTRODUCTION}

The question of the most suitable size of wire for the coil of a galvanometer, in order to secure a maximum sensitivity for certain definite conditions of use, arose during the development of the electric telegraph. Maxwell ${ }^{1}$ investigated the case of a moving-magnet galvanometer used to measure the current in an external circuit of resistance $R$, and concluded that for the maximum deflection per volt in the external circuit the galvanometer should be wound with wire of such size that its resistance will be $G$, where $G$ is defined by a relation which in the symbols of the present paper is

$$
G / R=D /(D+2 t)
$$

in which the right-hand member is the ratio of the diameter $D$ of the bare wire to the diameter over the insulation of thickness $t$. It

1 Marwell, Electricity and Magnetism, 2, sec. 716; 1873. 
was assumed that the wire was of uniform diameter throughout the coil.

Maxwell ${ }^{2}$ also investigated the best form of cross section for the circular coil of a moving-magnet galvanometer, and the manner in which the size of wire should be varied as the winding proceeded, in order to obtain the most efficient utilization of the winding space. Ayrton and Perry ${ }^{3}$ republished Maxwell's results and determined the condition for maximum sensitivity of a galvanometer having this most efficient form of winding. They found that the maximum sensitivity was obtained when the resistance of the galvanometer was equal to that of the external circuit. Heaviside ${ }^{4}$ examined Ayrton and Perry's analysis and demonstrated that the simplicity of their result did not contradict Maxwell's sensitivity theorem, nor did it depend on the particular form of cross section of the coil, nor on the law governing the variation of the diameter of the wire from layer to layer, but solely upon a different assumption which Ayrton and Perry had made, namely, that the thickness of the insulation was in every case to be a constant fraction of the diameter of the wire, or in present-day terms, that the space factor was to be constant.

An examination of Maxwell's analysis shows that the peculiar result he obtained (as given in equation (1)) depends upon his tacit assumption that $t$, the absolute thickness of the insulation, is constant for all sizes of wire. If in his preliminary equations $t$ be taken as a constant fraction of $D$, the result obtained by Ayrton and Perry will be obtained, namely, that for maximum sensitivity the galvanometer should be wound to have a resistance equal to that of the external circuit.

This theorem has often been quoted. For example, in the case of a wheatstone bridge having a resistance of $100 \mathrm{ohms}$ in each of the four arms the battery diagonal may be neglected for the condition of approximate balance, and, hence, the "internal resistance" of the bridge, which is the external resistance as far as the galvanometer is concerned, is $100 \mathrm{ohms}$. It is customary to say that in this case for maximum sensitivity one should choose a galvanometer having a $100-\mathrm{hm}$ coil if the choice lies between galvanometers which are identical in every respect except in size of wire, and, consequently, in number of turns and resistance of coil. While this advice can be followed with moving-magnet galvanometers, it will, in general, result in serious loss of time in taking readings if the galvanometers in question are of the moving-coil type without damping frames, because as a rule such galvanometers as now made are much overdamped when connected to an external circuit having a resistance equal to the coil resistance. It will, however, be convenient, in the analysis now to be presented, to assume initially that the galvanometers are either of the moving-magnet type, or that for other reasons considerations of damping may be neglected. The manner in which the conclusions reached may need to be modified for movingcoil galvanometers will be indicated in a later section of this article entitled "Damping Limitations of the Moving-Coil Galvanometer."

It is often the case, in the mathematical treatment of problems of maxima and minima, that no investigation is made concerning the manner of variation of the dependent variable on both sides of the

Heaviside, J. Soc. Teleg. Engrs., 9, pp. 202-206; 1880. 
point of maximum or minimum. Such incomplete treatment of the problem is apt to be very misleading, and one should not blindly adopt the optimum condition at the expense of other qualities without first investigating to see what happens for a considerable range of values of the independent variable on both sides of that which gives this optimum condition. ${ }^{5}$

The object of this paper is to show that while the theorems of Maxwell and of Ayrton and Perry concerning what may be called the working sensitivity of a galvanometer are theoretically correct, there is nevertheless a great deal of latitude in the choice of the resistance of the galvanometer, with only a relatively small resulting loss in sensitivity, and with some material advantages. The manner in which the user may take advantage of this fact will be indicated.

\section{SENSITIVITY OF THE MOVING-MAGNET GALVANOMETER}

There are various ways of expressing the sensitivity of a galvanometer, all of which ultimately relate to its current sensitivity; that is, its response (deflection) for unit current in its coil. There are cases, such as in the measurement of insulation resistance by the directdeflection method, where the external resistance is so high that the current sensitivity is the important property, but the scope of this paper is limited to cases where the resistance of the external circuit does not differ from that of the galvanometer by more than say a few orders of magnitude. For such cases the user is concerned with what might be called the "working microvolt sensitivity," but which will be called for brevity the "working sensitivity" in the following discussion. The working sensitivity applies only to the user's particular problem at the moment, and may be defined as the response of the galvanometer for unit electromotive force in a circuit which includes the galvanometer and a particular external circuit. As illustrations may be cited: (a) The measurement, by direct deflection, of a small thermal emf. set up in a thermocouple of given resistance; (b) the detection (and reduction to zero) of the small electromotive force across the galvanometer diagonal of an unbalanced wheatstone bridge.

The moving-magnet galvanometers in use in Maxwell's time had coils of circular form in which all portions of the length of any given turn of wire were equally effective in setting up a magnetic field at the center of the coil. For simplicity this form of galvanometer will be assumed initially in this treatment of the problem, and the case of the permanent-magnet moving-coil (d'Arsonval) galvanometer will be considered later.

- An example, although unrelated to the present case, will serve to demonstrate this point. Suppose that it is desired to make a metal "weight" in cylindrical form. It may easily be shown that to have the necessary volume of metal with the desirable minimum of exposed surface the diameter of the welght should equal its height. However, other considerations may call for a difforent form with the boight either much smaller or much greater than the diameter. It may be shown that when the diamoter is twice (or one-half) the height, the volume-surface ratio is only 5 per cent below its desirable maximurn value, and for the diameter equal to four times (or one-fourth) the height the ratio is still within 13 per cent of the maximum. It is obvious that one does not have to sacrifice much in other requirements to obtain virtually the full benefit of the opcimum form of weight.

That galvanometer makers and users do not realize how much latitude is possible is shown by statements in catalogues and text books. For example, a recent (1928) textbook on electrical measurements states that "In the choice of a galvanometer for a definite measurement one should be careful to have the resistance of the galvanometer as nearly as possible of the same order of magnitude as the resistance of the measurement circuit." An equally recent catalogue states: "It should be remembered that for a given constant electromotive force in circuit, the resistance of the coil should, If possible, equal all the resistance of the external circuit, including the suspension." 
Referring to the circular coil shown in cross section in Figure 1, let

$L=$ mean length of turn; that is, total length of wire divided by number of turns.

$D=$ diameter of bare wire.

$\rho=$ volume resistivity of material of wire, assumed constant.

$t=$ thickness of insulation.

$N=$ number of turns of wire in the coil.

$G=$ resistance of the coil.

$R=$ resistance of external circuit.

$E=$ electromotive force acting in the circuit.

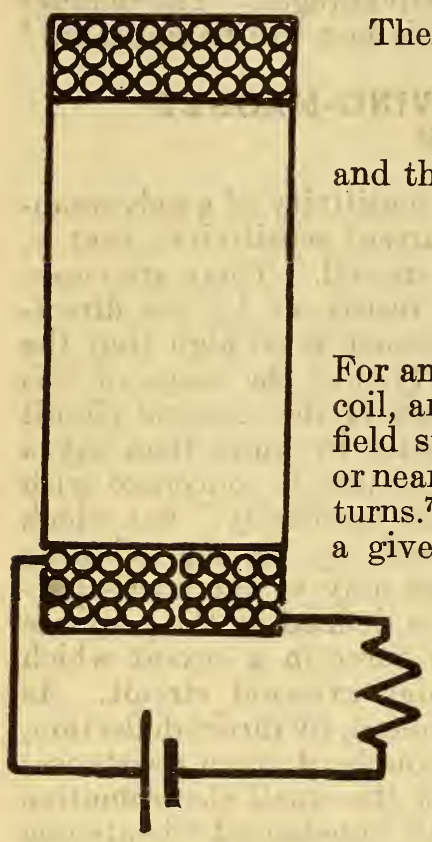

Figure 1.-Galvanometer coil connected to an external circuit containing an electromotive force

$$
G=4 N L \rho / \pi D^{2}
$$

and the current is

$$
\begin{aligned}
I & =E /(R+G) \\
& =E /\left(R+4 N L_{\rho} / \pi D^{2}\right)
\end{aligned}
$$

If we multiply both sides of equation (3) by $N$ and divide by $E$, the result will be an expression for the ampere turns per volt, which is directly proportional to the working sensitivity of the galvanometer for the case of the particular value of resistance $R$ in the external circuit. Denoting this quantity, which is a function of the size of the wire, by $F$, we have

$$
\begin{aligned}
F & =N I / E \\
& =N /(R+G) \\
& =N /\left(R+4 N L_{\rho} / \pi D^{2}\right)
\end{aligned}
$$

7 This is true for a coil of any form having a cross section which may be the same everywhere or may change in form and limensions from point to point in any manner, provided the current distribution over a plane intersecting the cross section anywhere is not changed when the number of turns is changed. For a constant value of ampere turns such a plane will obviously cut the same total current regardless of the number of turns. 
In this expression $N$ and $D$ are not only interdependent, but depend on $t$, the thickness of the insulation. We may eliminate $N$ and introduce $t$ by using the fact that the volume $V$ occupied by the winding is constant for all sizes of wire and thicknesses of insulation and is

from which

$$
\begin{gathered}
V=N L(D+2 t)^{2} \\
N=V /\left[L(D+2 t)^{2}\right]
\end{gathered}
$$

Substituting this value of $N$ in equation (4), and simplifying, we get a general expression for the ampere turns per volt, namely,

$$
F=\frac{1}{L R(D+2 t)^{2} / V+4 \rho L / \pi D^{2}}
$$

in which the only variables are the diameter of the bare wire $D$, and the thickness of insulation $t$. Such a general relation would be represented by a surface. Since in wire-manufacturing practice there are fairly definite relations already established between the diameter $D$ and thickness of insulation $t$, we may make $F$ a function of a single variable by making $t$ a constant or by introducing a suitable relation between $D$ and $t$. For example, in the case of silk-covered wire the thickness of the insulation is usually constant regardless of the diameter of the bare wire. Differentiating equation (7) on this basis, we have

$$
\frac{d F}{d D}=-\frac{2 L R(D+2 t) / V-8 \rho L / \pi D^{3}}{\left[L R(D+2 t)^{2} / V+4 \rho L / \pi D^{2}\right]^{2}}
$$

The condition giving a maximum value of $F$ is obtained by equating the numerator of the right-hand member of equation (8) to zero, from which

that is

$$
\begin{aligned}
& 4 \rho / \pi D^{3}=R(D+2 t) / V \\
& 4 \rho V /\left[\pi D^{3}(D+2 t)\right]=R
\end{aligned}
$$

which on substituting the value of $V$ from equation (5) and that of $G$ from equation (2) becomes

or

$$
G(D+2 t) / D=R
$$

$$
G / R=D /(D+2 t)
$$

which is identical with the expression found by Maxwell for the case of $t$ constant for all sizes of wire.

As a second relation, which may be taken to hold with sufficient approximation for the sizes of enamel-insulated wire used in the construction of moving-coil galvanometers, the thickness of the insulation may be assumed to be directly proportional to the diameter of the bare wire; that is,

$$
t=a D
$$

When this value of $t$ is substituted, equation ( 7 ) becomes

$$
F=\frac{1}{L R D^{2}(1+2 a)^{2} / V+4 \rho L / \pi D^{2}}
$$


from which

$$
\frac{d F}{d D}=-\frac{2(1+2 a)^{2} L R D / V-8 \rho L / \pi D^{3}}{\left[L R D^{2}(1+2 a)^{2} / V+4 \rho L / \pi D^{2}\right]^{2}}
$$

Equating the numerator to zero we have the condition for a maximum value of the function $F$ for this case, namely,

$$
4 \rho / \pi D^{3}=(1+2 a)^{2} R D / V
$$

from which

$$
4 \rho V /\left[\pi D^{4}(1+2 a)^{2}\right]=R
$$

Substituting for $V$ its value $N L D^{2}(1+2 a)^{2}$, there results

$$
4 N L \rho / \pi D^{2}=R
$$

that is

$$
G=R
$$

which is the result found by Ayrton and Perry after introducing the condition that the thickness of the insulation bears a constant ratio to the diameter of the bare wire.

For the sake of having a simple form of coil in mind the preceding analysis has been referred to the simple circular coil of rectangular cross section shown in Figure 1. However, the conclusions are applicable to a galvanometer having a coil of any form with a cross section which may be either of the same form everywhere or may change from point to point. This follows from the fact already stated, preceding the writing of equation (4), namely, that the magnetic field at any point within or near a coil of any form whatever will depend solely upon the ampere-turns regardless of the number of turns in the coil.

\section{SENSITIVITY OF THE MOVING-COIL GALVANOMETER}

In the preceding discussion every portion of the length of the wire composing the galvanometer coil was effective in setting up a magnetic field at the center of the coil upon which the deflection depends. In the permanent-magnet moving-coil (d'Arsonval) galvanometer, which is the favorite instrument at the present time for all but very special work where the sensitivity must be pushed to extreme values, the galvanometer resistance is made up of three parts; $(a)$ that of the elements of the coil which may be considered as active, that is, those parts of the vertical sides which cut the flux of the permanent magnet and provide the torque; $(b)$ that of the top and bottom parts of the coil which do not cut the flux and which may be considered as serving merely to connect the active wires in series; $(c)$ the resistance of the suspensions. ${ }^{8}$ Since the suspensions are assumed to remain unchanged as the size of wire in the coil is changed, their resistance may be taken as constant. Obviously, this resistance should be counted in as part of the external circuit. This procedure is particularly important for galvanometers having coils with a resistance of only a few ohms, in which case the resistance of the suspensions may be several times the

- Many galvanometers of the moving-coil type have the moving coil supported by pivot-and-jewel bearings, and have spiral springs which serve the dual purpose of carrying current to and from the coil and of supplying the counter force. For example, many pyrometer galvanometers used with thermocouples are of this description. It will be understood that whenever the word "suspension" is used in this article the use of spiral springs as an electrical and mechanical equivalent is understood 
resistance of the coil. It might seem at first glance that the resistance of the "inert" horizontal wires at the top and bottom of the coil ought also be counted as part of the external resistance, but it will be shown that this is not the case.

In order that the preceding reasoning for the moving-magnet galvanometer may be applied to the moving-coil galvanometer it is only necessary to show that in the latter the torque varies directly as the ampere turns in the coil, just as in the former the magnetic field at the center of the coil, and, consequently, the torque on the moving magnet varies as the ampere turns. In the moving-coil galvanometer the torque $T$ is equal to the product of four factors; that is

$$
T=H \cdot I \cdot(2 N h) \cdot b / 2
$$

in which $H$ is the magnetic field intensity in the air gap, $I$ the current, $2 N h$ the total length of active wire, made up of two vertical sides of each of the $N$ turns of effective height $h$, and $b / 2$ is one-half the effective breadth of the coil; that is, it is the average distance from the individual vertical wires to the axis of rotation of the coil. Equation (14) may be written

$$
\begin{aligned}
T & =H h b \times N I \\
& =\text { Constant } \times \text { ampere turns }
\end{aligned}
$$

In this expression the coefficient of $N I$ is a constant for the given galvanometer regardless of the size of the wire of which the coil is wound. Hence, all the relations previously derived for the movingmagnet galvanometer apply to the moving-coil galvanometer also, if the resistance of the suspensions be included as part of the external resistance.

\section{SENSITIVITY FOR CONDITIONS WIDELY DIFFERENT FROM THE OPTIMUM}

It is not possible to obtain stock galvanometers in closely graded steps of coil resistance and it would be necessary to draw special sizes of wire to produce them, as may be seen from the fact that in a series of coils of constant space factor wound from consecutive A. W. G. sizes of wire the resistances of any two adjacent coils in the series would be in the ratio of 1.6 to 1 . It will be of interest, therefore, to see what happens when the resistance of the galvanometer varies from a value very much lower than the optimum value to one very much higher, and having thus discovered how much latitude is possible without serious loss of sensitivity, to consider in what way this latitude may be turned to practical advantage. In order to do this conveniently, a relation will be developed from the preceding equations, as follows:

The general expression for the function, ampere turns per volt, which is proportional to the working sensitivity, is

$$
F=N /(R+G)
$$

This relation holds without restriction as to the ratio of the diameter of the wire to the thickness of insulation. From this point on one must select some definite relation between these quantities.

$$
88500^{\circ}-30-9
$$


Taking the case of thickness of insulation directly proportional to wire diameter; that is, a constant space factor, from equations (4) and (13) we have that

$$
F_{\max }=N_{1} / 2 R
$$

where $N_{1}$ denotes the particular number of turns in the coil of resistance $G_{1}=R$, which gives maximum sensitivity when used with an external circuit of resistence $R$. Dividing equation (4) by equation (16),

$$
\begin{aligned}
\frac{F}{F_{\max }^{\prime}} & =\frac{N}{R+G} \cdot \frac{2 R}{N_{1}} \\
& =\frac{2 R}{R+G} \cdot \frac{N}{N_{1}}
\end{aligned}
$$

Since the resistances of two coils wound in identical winding spaces, using wires of the same space factor, are to each other as the squares of their numbers of turns,

$$
N^{2} / N_{1}^{2}=G / G_{1}
$$

and since by equation (13)

it follows that

$$
G_{1}=R
$$

$$
N / N_{1}=\sqrt{G / R}
$$

Introducing this value into equation (17), it becomes

$$
\frac{F}{F_{\max }}=\frac{2}{\sqrt{G / R}+\sqrt{R / G}}
$$

which can also be written in a form more convenient for computation namely,

$$
\frac{F}{F_{\max }^{\prime}}=\frac{2}{\sqrt{G / R}+1 / \sqrt{G / R}}
$$

After the first term of the denominator has been computed its reciprocal gives the second term. This function has the value unity when $G=R$. It is plotted as curve $A$ in Figure 2 , in which the ordinates are values of $F / F_{\max }$ plotted in per cent of maximum sensitivity, and the abscissas are values of the ratio of galvanometer resistance to external resistance. The curve has such a flat maximum that it is necessary to plot the abscissas to a logarithmic scale in order to show the behavior of the function over an adequate range of relative values of galvanometer resistance to external resistance. ${ }^{9}$

As an example of the use of curve A (fig. 2) let the external resistance be $100 \mathrm{ohms}$, so that a $100-\mathrm{ohm}$ galvanometer is indicated as the most sensitive. It may be seen that one can use a galvanometer of any resistance between $20 \mathrm{ohms}$ and $500 \mathrm{ohms}$ and still obtain a sensitivity not less than 74 per cent of the maximum.

9 After this paper was written the author discovered that an equation identical in substance with equation (18) had been given by Schuster in a paper entitled "Electrical Notes," in Phil. Mag. 39, p. 175, 1895. Although Schuster does not so state, his reasoning applies only to galvanometers wound with wires of a constant space factor. In view of the fact that the useful information given by Schuster seems to have escaped general attention, possibly because of the rather indefinite title of the paper, it appears desirable to point out again the relationships involved in greater detail and to extend the treatment to the case of a constant thickness of insulation as exemplified by silk-covered wire. 


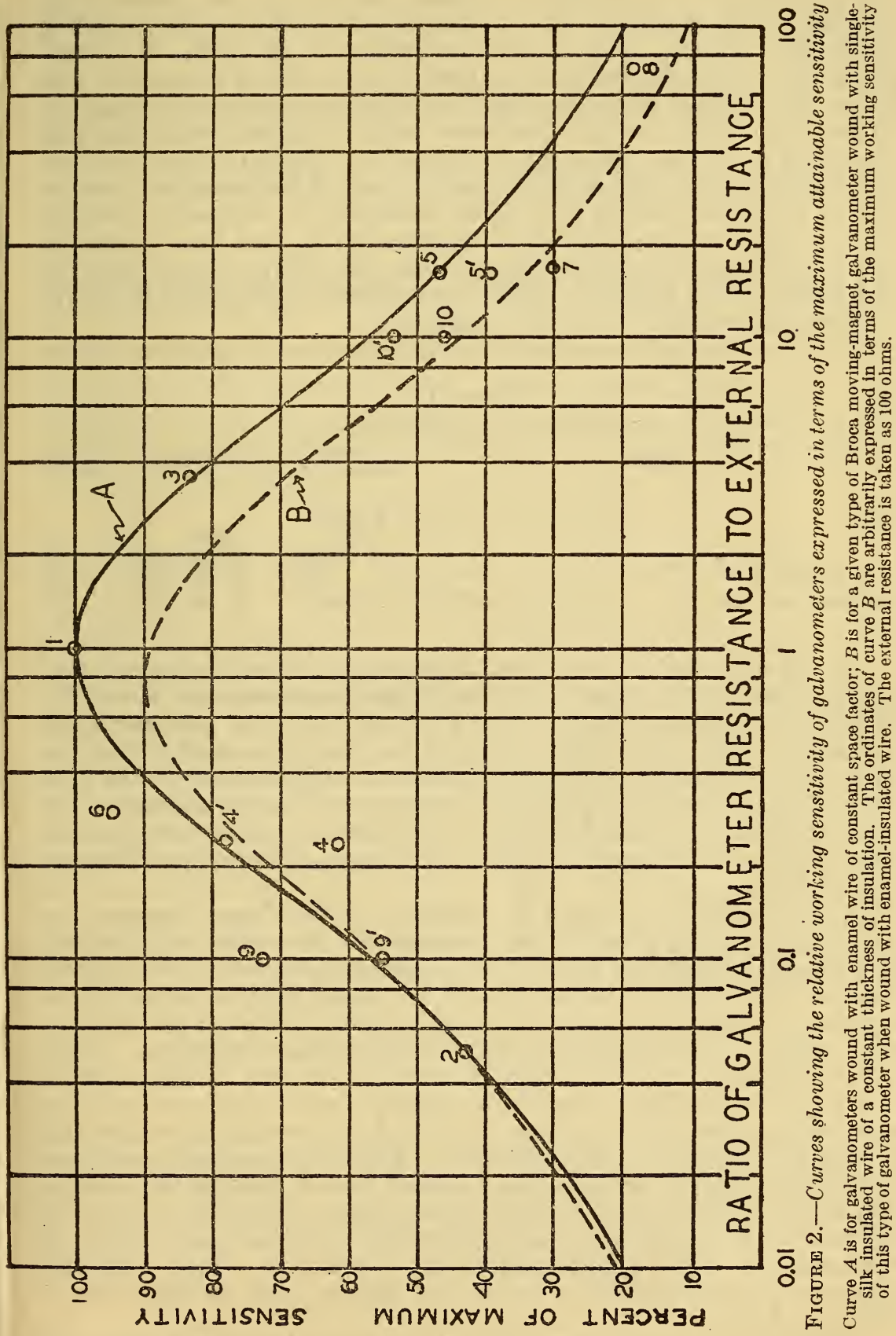


The plotted points of curve A (fig. 2) represent computed values of relative sensitivity of actual galvanometers for which representative average values of coil resistance, current sensitivity, etc., were kindly supplied by the makers. Four groups of galvanometers are included, nominally differing only in the resistance of the coil. For each group one galvanometer, of an intermediate value of resistance, was taken as a basis of reference, and its deflection for a given voltage in an external circuit having a resistance equal to its own resistance was plotted as point No. 1, namely, 100 per cent. Point 1 is thus common to all the groups. The deflection which each of the other galvanometers of the group would give when connected to the same external circuit with the same electromotive force acting was then computed as a percentage of the deflection given by the reference galvanometer and plotted as a point for which the abscissa is the ratio of galvanometer resistance to external resistance.

The following table gives particulars concerning the galvanometers:

TABLE 1 .

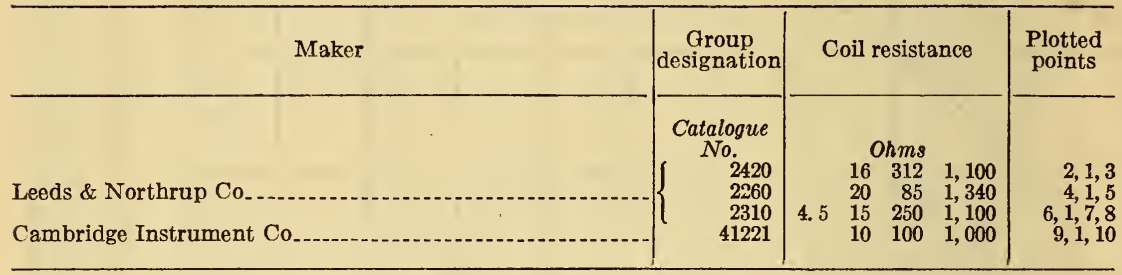

The galvanometers of the first three groups are of the permanentmagnet moving-coil type. The Cambridge galvanometers are of the Broca moving-magnet type. The Leeds \& Northrup galvanometers are wound with enamel-insulated wire for which the thickness of the enamel is very nearly a constant fraction of the diameter of the bare wire. The coils of the Cambridge Instrument Co. galvanometers are made in England, and it has been assumed in this paper that the wire used in them has a constant ratio of thickness of enamel to diameter of bare wire.

In the manufacture of galvanometers it is not found necessary to adjust the strength of individual magnets and the torsion of individual suspensions to standard values. It is sufficient to control these quantities so that at least a certain stated minimum value of sensitivity is attained. The failure of some of the points of Figure 2 to lie on or near curve $\mathrm{A}$ is the result of these manufacturing variations. From the maker's data on the number of turns in the coils and the current sensitivity it has been possible to compute some values of relative sensitivity corrected to the basis of a standard strength of magnet and a standard torsion of the suspension. The corresponding points are denoted on Figure 2 by primed numerals; thus $4^{\prime}$ denotes the relative sensitivity of catalogue No. 2260 galvanometer of $20 \mathrm{ohms}$ resistance reduced to the basis of the same strength of magnet and torsion of suspension as the 85-ohm galvanometer of this type, which is the reference galvanometer of this group; and similarly for the other points $5^{\prime}, 9^{\prime}$, and $10^{\prime}$.

Although the greater space factor of the enamel-insulated wire is an important advantage in the case of moving-coil galvanometers, particularly for the finer sizes of wire, there are instances where single-silk 
insulation is used. For moving-magnet galvanometers of relatively low resistance (say $10 \mathrm{ohms}$ or less) the space factor of the single-silk and the enamel-insulated wires may be so nearly equal that other considerations may determine the choice. The relative working sensitivity of galvanometers wound to have various resistances, using wire with a constant thickness of insulation, will now be discussed. It is assumed that the galvanometers are identical in all respects other than the size of wire in the coil.

Returning to equation (9), and denoting by $D_{1}$ and $G_{1}$ the particular values of $D$ and of $G$ which give maximum sensitivity, we have

$$
G_{1} / R=D_{1}\left(/ D_{1}+2 t\right)
$$

The length of wire in the coil, the diameter of the wire over the insulation being $D_{1}+2 t$, is

$$
N_{1} L=V /\left(D_{1}+2 t\right)^{2}
$$

where $N_{1}$ is the particular value of $N$ corresponding to $D_{1}$. The resistance of the coil is obtained by multiplying this value of the length by $\rho$ and dividing the product by the cross-sectional area of the conductor; that is,

$$
G_{1}=4 V \rho /\left[\pi D_{1}^{2}\left(D_{1}+2 t\right)^{2}\right]
$$

Substituting this value of $G_{1}$ in equation (20) and simplifying,

$$
D_{1}{ }^{4}+2 t D_{1}{ }^{3}=4 V \rho / \pi R
$$

which is not a general equation, but holds only for the diameter of wire giving maximum sensitivity.

While a solution of equation (23) by analytical methods is possible, the procedure is laborious, and for practical purposes a graphical method is much simpler and quicker. The curve (fig. 3) has as abscissas values of optimum diameter of bare wire $D_{1}$, and as ordinates values of the function $D_{1}^{4}+2 t D_{1}^{3}$, using for $2 t$ its usual value for single-silk covering, namely, 0.002 inch $(0.05 \mathrm{~mm}$.). For a given external resistance $R$, and a series of galvanometers which differ only in the resistance of the coil, that is, only in the diameter $D$ of the bare wire, the value of the quantity $4 V \rho / \pi R$, the right-hand member of equation (23) can be computed. The value of the abscissa $D_{1}$ of the curve (fig. 3) corresponding to this value of $4 V \rho / \pi R$ as ordinate can then be found directly.

Returning to the general expression for $F$, the ampere turns per volt, to which the working sensitivity is proportional, namely,

$$
F=N /(R+G)
$$

using the symbols $F_{\max }, N_{1}$, and $D_{1}$ in the same sense as in the preceding discussion, and expressing the coil resistance $G$ in terms of $R$ and $D_{1}$ by equation (9), it may be shown by an analysis similar to the preceding that the equation for the relative sensitivity for the case of a constant thickness of insulation takes the form

$$
\frac{F}{F_{m a x}}=\frac{2 R}{R+4 V \rho /\left[\pi D^{2}(D+2 t)^{2}\right]} \cdot \frac{\left(D_{1}+t\right)\left(D_{1}+2 t\right)}{(D+2 t)^{2}}
$$

Having found the value of $D_{1}$ for any given case by using the abovementioned curve, the right-hand member of equation (24) contains only one variable, $D$. Values of $F / F_{\max }$ may then be computed for a 
number of values of $D$ above and below the particular value $D_{1}$ for maximum working sensitivity, and a curve may be plotted giving

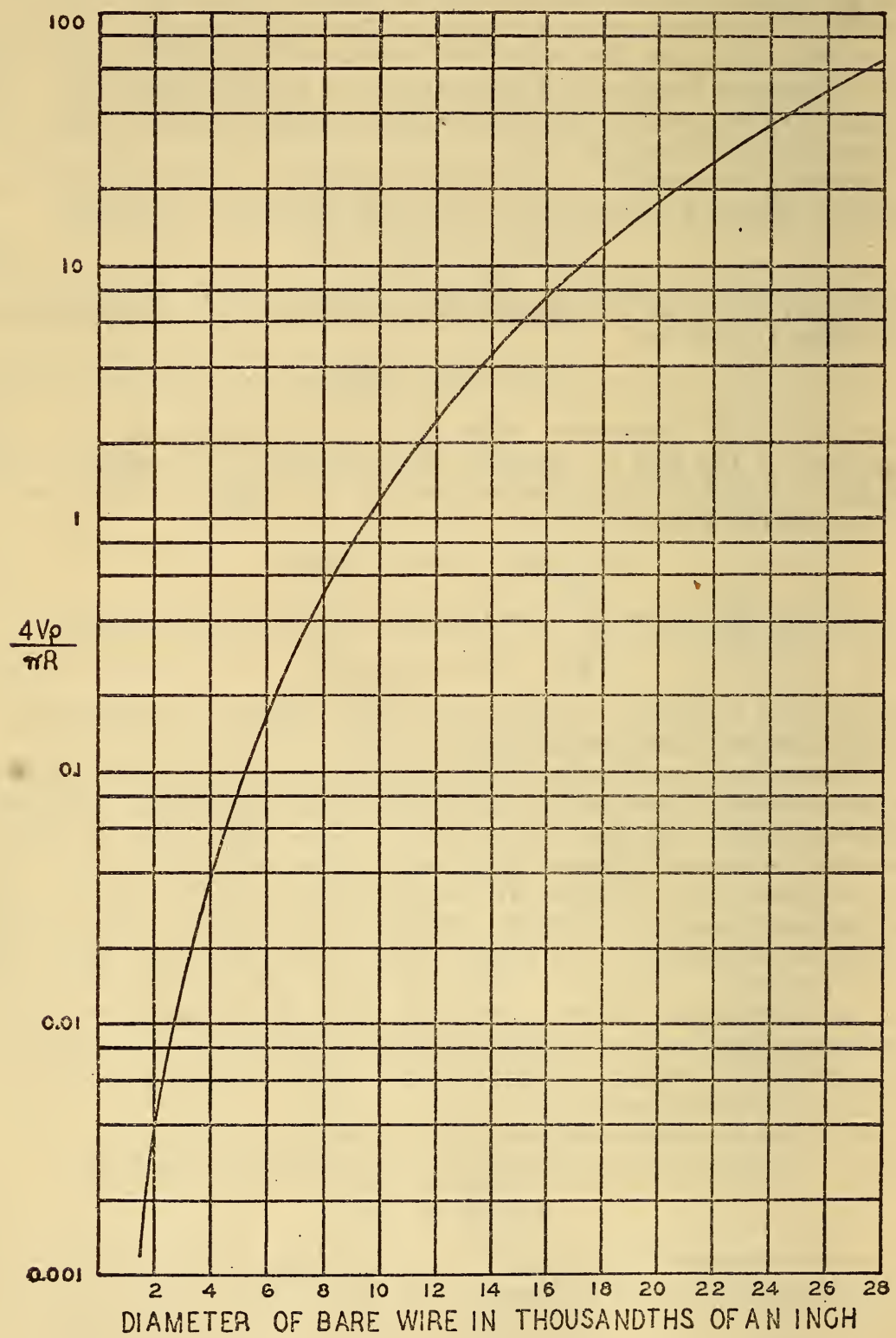

Frgure 3.-Curve for the graphical solution of the biquadratic equation (23) for the optimum diameter of wire for the case of a constant thickness of insulation, namely, 0.001 inch

For a given winding volume $V$ (cubic inches), resistivity $\rho$ (numerically equal to the resistance between opposite faces of a 1-inch cube) and external resistance $R$ ohms, the curve is entered with the product $4 V \rho / \pi R$. The corresponding ordinate has as alsscissa the diameter of the wire.

values of relative sensitivity $F / F_{\text {max }}$ as a function of the coil resistance . $4 V \rho /\left[\pi D^{2}(D+2 t)^{2}\right]$, values of which for the various chosen values 
of $D$ being obtained during the process of computing the values of $F / F_{\max }$ from equation (24).

Curve B (fig. 2) has been computed for Cambridge Instrument Co. Broca galvanometers on the assumptions that the coils are wound with single-silk insulated copper wire and that the external resistance is $100 \mathrm{ohms}$. Comparing the two curves of Figure 2 it is seen that the maximum working sensitivity, using silk-covered wire, would be about 90 per cent of the maximum for the 100 -ohm Broca galvanometer as it is actually wound with enamel-insulated wire. The maximum working sensitivity, using silk-covered wire, occurs when the galvanometer resistance is a little over $80 \mathrm{ohms}$ as required by Maxwell's theorem, equation (1). The two curves intersect when the ratio of galvanometer resistance to external resistance is 0.062 ; that is, when the resistance of the galvanometer is $6.2 \mathrm{ohms}$ for either kind of insulation. This is the case where the silk and the enamel have the same thickness. For galvanometers of lower resistance curve $B$ lies a little above curve $\AA$, which means that for the coarse wires used in these coils the silk is thinner than the enamel. ${ }^{10}$

Going to the right of this point of intersection the ratio of the working sensitivity of the galvanometer wound with silk-covered wire to that of the galvanometer of the same resistance having enamelinsulated wire decreases continuously, reaching the value 0.54 when the galvanometer resistance is $10,000 \mathrm{ohms}$.

\section{DAMPING LIMITATIONS OF THE MOVING-COIL GALVANOMETER}

Although the effect of the resistance of the external circuit on the damping is of little or no consequence in the use of moving-magnet galvanometers it is a very important matter when galvanometers of the moving-coil type are concerned. In these latter the following cases arise in practice:

(a) The moving coil may be wound on a closed metal frame, or may be provided with a second closed damping winding which has no connection to the external circuit. One form of this auxiliary winding consists of a single closed turn of copper wire, and is sometimes spoken of as a "damping rectangle." The use of this expedient in any form makes it possible to construct (or alter) a galvanometer so that the external critical damping resistance may have any desired value (above a certain minimum), even infinity; that is, the motion of the coil may be made aperiodic when its terminals are not joined by any conducting path. A well-designed direct-current voltmeter is a common example of this case. In it the moving coil is wound on an aluminum frame which provides nearly all the necessary damping.

(b) The damping winding or rectangle of plan (a) has the disadvantage that, being of service only while the coil is in motion, it adds otherwise needless weight and moment of inertia to the moving system. Consequently, when high sensitivity and shortness of period are desired, it is customary to avoid the use of damping coils, in which case the kind of motion of the coil and, therefore, the time required to obtain a reading depend very much on the value of the external

10 This is on the assumption that the thickness of the enamel is actually a constant fraction of the diameter of the wire. While this is very nearly true for the sizes of wire used in the moving-coil galvanometers of Table 1, the practice of various makers of enamel-insulated wire is not uniform. 
resistance. Many users prefer a slight amount of underdamping, such that the coil passes the point of final deflection by a small amount and then returns to this point without further observable oscillation. This slight overshoot gives the assurance that neither overdamping nor friction ("sticking") is present.

One point of considerable importance to the galvanometer user is that if the criterion be the time required to obtain a reading a much wider tolerance is possible for the case of underdamping. This may be illustrated by the results of an experiment made on a pivoted pointer galvanometer having a frameless coil. The period of oscillation on open circuit was 2.6 seconds and the time to come to apparent rest when critically damped 3.4 seconds. As the total resistance was reduced below the value for critical damping, the time to come to rest increased sharply, reaching a value of 8 seconds when the total resistance was two-thirds of the critical value. On increasing the resistance beyond the critical value the time to come to rest became 5,6 , and 8 seconds for a total resistance twice, three times, and four times the critical value. ${ }^{11}$

The important conclusion to the galvanometer user from this experiment is that if he must choose between underdamping and overdamping the former will give him much greater latitude in the total resistance for a given limit on the time to obtain a reading. Of this total resistance the external resistance is the part with which the user is usually more concerned. It sets a limit to the "internal resistance" of the bridge, potentiometer, thermocouple, or other device to which the galvanometer is connected. Assuming that critical (or slight under) damping is required the user who has already acquired a given galvanometer is usually concerned very little ${ }^{12}$ about the resistance of its coil, but very much concerned with the value of external critical damping resistance. If the resistance of the apparatus to be connected to the galvanometer is less than the given value of external critical damping resistance a rheostat in series with the apparatus may be used to bring up the external resistance to its value for critical damping. The working sensitivity will thus be constant for all values of apparatus resistance from zero to the value for critical damping. If the resistance of the apparatus is greater than the external critical damping resistance, the galvanometer must be shunted to obtain critical damping, and it may be shown that if the external circuit has a resistance $n$ times the critical value and the galvanometer shunt has the proper resistance to produce critical damping, the working sensitivity will be reduced to $1 / n$ of its former value. ${ }^{13}$

If, however, a galvanometer is to be selected from a number which differ structurally only in size of wire and number of turns in the coil, and, consequently, differ electrically in coil resistance and external critical damping resistance, the analysis in the earlier

\footnotetext{
11 Such a result follows from the laws governing the motion of a galvanometer. The figures given in the above example should not be construed to apply generally because one must decide on a definition of "coming to rest." For example, if the motion of the coil is exactly aperiodic it reaches its theoretical rest position only after an infinite time. However, it reaches this position, within 0.1 per cent of the defiection, in about $1.5 T$, where $T^{\prime}$ is the undamped free period. For underdamped and overdamped motion also the coil theoretically never reaches a condition of absolute rest.

12 An exception to this statement must be made when one is concerned with the effect of ambient temperature variations on the indications of a galvanometer which is connected to an external device; for example, a thermocouple. In this case there is a particular value of the temperature coefficient of resistance of the entire circuit which will offset the temperature coefficient of torsional rigidity of the suspension. The high temperature coefficient of resistance of the galvanometer coil makes the resistance of the coil a matter of importance if independence of variations of ambient temperature is sought.

${ }_{13}$ This is shown graphically for a particular numerical example by fig. 1, p. 224, of a paper by Wenner on General Design of Critically Damped Galvanometers; Bull. Bur. Stds., 13, 1916; Scientific Paper No. 273.
} 
part of this paper shows, first, that one can to a large extent ignore the traditional statement concerning the condition for maximun working sensitivity, and being free from this supposed limitation may select a galvanometer having an external critical damping resistance most nearly equal to the resistance of the apparatus with which it is to be used. As moving-coil galvanometers are now made this would usually mean choosing a galvanometer having a net coil resistance of say from one-third to one-tenth of the resistance of the connected apparatus. Such a galvanometer will require a minimum amount of reduction of sensitivity to effect the desired kind of damping and may easily have several times the working sensitivity of a similar galvanometer chosen according to the traditional rule, but requiring an excessive amount of series resistance to effect the desired damping.

It may be pointed out that for some applications a heavily overdamped galvanometer is desired in order to smooth out transitory fluctuations and give an average value.. The preceding analysis shows that for this case also one may largely disregard the traditional rule and select a galvanometer having a net coil resistance several times the resistance of the external apparatus, and thus secure the desired overdamping with relatively small loss in working sensitivity. In so doing it should be kept in mind that the effect of changes of room temperature will be relatively large. If independence of roomtemperature variations is necessary, the resistance of the galvanometer coil must be only a fraction ${ }^{14}$ of the total resistance and the necessary overdamping must be obtained by winding the coil on a damping frame.

The use of a damping frame, coil, or rectangle (case $(a)$ ) is usually confined to the original construction of the galvanometer and does not lend itself readily to subsequent alterations. Properly applied it makes it possible to produce galvanometers which have a very large ratio of total critical damping resistance to coil resistance. Such galvanometers are usually of high current sensitivity and are suitable for work (such as insulation testing by the direct-deflection method) where the external resistance will be so large that the ordinary coil damping is negligible.

\section{CONSTRUCTIONAL LIMITATIONS}

The theoretical treatment of the problem in this paper has neglected for generality, certain practical limitations which operate to modify the results obtained in actual galvanometers. It is probable that the maker usually limits himself to wires of certain standard diameters with corresponding standard thicknesses of insulation. There must be an integral number of turns per layer, and (what may be more important) an integral number of layers in the coil. In consequence, when a particular size of wire is used the theoretical number of layers for the assigned radial depth of winding might be, say, 4.6. In this case 4 layers will have to be used if 5 would cut down the clearances between the coil and the pole pieces and core below the permissible minimum. It will, therefore, be realized that in checking the performance of a line of galvanometers which nominally differ only in resistance some variation from the theoretical performance must be expected, especially for coils of low resistance made of coarse wire. 
The maker sometimes intentionally weakens the magnet of one or more galvanometers of a given series, all nominally alike except as to resistance, in order to get for these exceptional ones particular values of external resistance for critical damping. Also, in some groups of moving-coil galvanometers the volume of wire composing the coil varies greatly within the group, the tendency being to use larger volumes for the coils of finer sizes of wire, for which the microampere sensitivity is the important feature. In such cases one can not expect the performance to follow a law which includes the assumption of identical volumes of wire in the coils.

If galvanometers are nominally alike, except as to resistance and period, the values of their current sensitivities should be reduced to the basis of a common period before their working sensitivities are compared by the method given in this paper. This reduction is made by using the relation that when other things are equal the deflection per microampere increases as the square of the period.

\section{PRACTICAL CONSIDERATIONS}

Some of the practical advantages which may be derived from the very flat maxima of the relations shown by the curves of Figure 2 are as follows:

(a) The galvanometer maker can restrict the number of standard windings to comparatively few and still meet nearly all ordinary requirements.

(b) The user can purchase galvanometers of a correspondingly small number of values of resistance, which values may be widely different, with the knowledge that the galvanometers will serve for all but extraordinary needs which require the construction of special galvanometers.

(c) Since the user has so much latitude in the matter of galvanometer resistance he can select the one having a value of external resistance for critical damping most nearly equal to the actual external resistance. This reduces to a minimum the time required to obtain a reading, and conserves a certain amount of sensitivity that might otherwise be lost by the use of shunt or series resistance to obtain proper damping. This consideration of proper damping is a very important one and will often govern the choice.

The function expressed by equation (18) and plotted as curve A in Figure 2 has an interesting property which is evident from the form of the right-hand member of the equation, namely, that the same value of relative sensitivity $F / F_{\max }$ will result for any given ratio $G / R$ or for its reciprocal $R / G$. That is, if a given percentage of the maximum sensitivity is obtained when the resistance of the galvanometer coil is $m$ times the resistance of the external circuit, the same percentage will be obtained when the galvanometer resistance is $1 / \mathrm{m}$ times the external resistance. Since galvanometers of lower resistance, being wound with coarser wire, are less liable to damage by accidental overload, and have some other advantages, such as higher microvolt sensitivity when used with bridges of low resistance, one would naturally choose galvanometers of lower resistance and equal working sensitivity, except as the consideration of damping might prevent.

Washington, August 21, 1929. 